\title{
Infecciones y coinfecciones respiratorias: patrón geográfico y de circulación poblacional
}

Norvell Perezbusta-Lara, Rocío Tirado-Mendoza* y Javier R. Ambrosio-Hernández

Universidad Nacional Autónoma de México, Facultad de Medicina, Departamento de Microbiología y Parasitología, Ciudad de México, México

\section{Resumen}

Introducción: Las infecciones respiratorias agudas constituyen la segunda causa de mortalidad en los niños menores de cinco años, con 150.7 millones de episodios anuales. Entre los principales agentes etiológicos están Orthopneumovirus (hOPV) y metapneumovirus (hMPV) humanos como primera y segunda causa de bronquiolitis, respectivamente; Orthorubulavirus humano tipo 2 (hORUV) se ha asociado a neumonía en pacientes inmunocomprometidos. Objetivo: Definir patrones de distribución geográfica y de circulación de hOPV, hMPV y hORUV. Método: Se llevó a cabo un estudio piloto transversal prospectivo observacional. Se genotipificaron 200 aislamientos virales de pacientes pediátricos mediante transcripción inversa seguida de reacción en cadena de la polimerasa en punto final (RT-PCR). Resultados: Se tipificaron 186 muestras positivas: 84 de hOPV, 43 de hMPV, dos de hORUV y 57 de coinfecciones. Se trazó la distribución geográfica. Las incidencias acumuladas de hMPV, hOPV y hORUV fueron de 0.215, 0.42 y 0.01, respectivamente. Las incidencias acumuladas de la coinfección de hMPV-hORUV y hMPV-hOPV fueron de 0.015 y 0.23; de hOPV-hMPV-hORUV, de 0.035; y de hORUV-hOPV, de 0.005. El mayor número de casos positivos de virus circulantes o cocirculantes se presentó entre enero y marzo. Conclusiones: Fue posible identificar patrones de circulación y distribución geográfica de los diferentes virus, así como de las coinfecciones virales.

PALABRAS CLAVE: Infecciones respiratorias agudas. Virus respiratorios humanos. Coinfecciones virales. Patrones de circulación.

\section{Respiratory infections and coinfections: geographical and population patterns}

\section{Abstract}

Introduction: Acute respiratory infections are the second cause of mortality in children younger than five years, with 150.7 million episodes per year. Human orthopneumovirus (hOPV) and metapneumovirus (hMPV) are the first and second causes of bronchiolitis; type 2 human orthorubulavirus (hORUV) has been associated with pneumonia in immunocompromised patients. Objective: To define hOPV, hMPV and hORUV geographical distribution and circulation patterns. Method: An observational, prospective cross-sectional pilot study was carried out. Two-hundred viral strains obtained from pediatric patients were genotyped by endpoint reverse transcription polymerase chain reaction (RT-PCR). Results: One-hundred and eighty-six positive samples were typed: 84 hOPV, 43 hMPV, two hORUV and 57 co-infection specimens. Geographical distribution was plotted. hMPV, hOPV, and hORUV cumulative incidences were $0.215,0.42$, and 0.01 , respectively. Cumulative incidence of $h M P V$-hORUV and hMPV-hOPV coinfection was 0.015 and 0.23; for hOPV-hMPV-hORUV, 0.035; and for hORUV-hOPV, 0.005. The largest number of positive cases of circulating or co-circulating viruses occurred between January and March. Conclusions: This study successfully identified circulation and geographical distribution patterns of the different viruses, as well as of viral co-infections.

KEY WORDS: Acute respiratory infections. Human respiratory viruses. Viral coinfections. Circulation patterns.

Correspondencia:

*Rocío Tirado-Mendoza

E-mail: tiradom@yahoo.com
Gac Med Mex. 2020;156:265-272

Disponible en PubMed

www.gacetamedicademexico.com

0016-3813/@ 2020 Academia Nacional de Medicina de México, A.C. Publicado por Permanyer. Este es un artículo open access bajo la licencia CC BY-NC-ND (http://creativecommons.org/licenses/by-nc-nd/4.0/). 


\section{Introducción}

Entre sus objetivos, la Organización Mundial de la Salud busca disminuir en dos terceras partes la mortalidad en menores de cinco años, que actualmente asciende a 8.8 millones por año; $37 \%$ ocurre en el primer mes de vida y $43 \%$ se asocia a enfermedades infecciosas, entre ellas la neumonía, responsable de $14 \%,{ }^{1}$ con 1.9 millones de muertes y 156 millones de episodios anuales en el mundo. ${ }^{2-4}$

Las enfermedades respiratorias agudas (IRA) son una de las principales causas de morbimortalidad, tanto en el neonato como en el lactante; ${ }^{5}$ entre los agentes etiológicos se encuentran bacterias y virus, como Orthopneumovirus humano tipo 2 (hOPV), Orthorubulavirus humano (hORUV) y virus emergentes como metapneumovirus humano (hMPV). ${ }^{6} \quad \mathrm{La}$ Organización Mundial de la Salud estima que aproximadamente $25 \%$ de las hospitalizaciones en los niños se debe a infecciones respiratorias agudas. El hOPV ${ }^{7}$ es responsable de $80 \%$ de este tipo de infecciones, seguido de hMPV. Estos agentes circulan en la población pediátrica todo el año, sin embargo, de diciembre a febrero se presenta un incremento en la incidencia de patologías respiratorias de origen infeccioso en menores de cinco años. Los recién nacidos prematuros, con asma, cardiopatías congénitas o displasia broncopulmonar son más susceptibles a esas infecciones, cuya presentación clínica suele ser más severa en comparación con la observada en niños sin comorbilidades. ${ }^{8}$

En México, las infecciones agudas del tracto respiratorio inferior constituyen la primera causa de morbilidad infantil por enfermedades infecciosas y se reportan entre las 10 primeras causas de muerte en niños entre uno y cuatro años de edad..$^{9,10}$ El diagnóstico se formula de acuerdo con la guía de práctica clínica, en la cual se describe que estas infecciones cursan frecuentemente con tos, sibilancias, estertores, dificultad para respirar y, en algunos casos, falla respiratoria que provoca la muerte. ${ }^{11}$

A pesar del impacto de estas infecciones en el orbe, en México no se cuenta con una red de vigilancia epidemiológica que brinde la información sobre los patrones de circulación de estos virus, la incidencia de coinfecciones virales ni su impacto en las manifestaciones clínicas. ${ }^{12}$ Las coinfecciones virales se asocian a un aumento en la morbimortalidad infantil y provocan cuadros "atípicos", lo que dificulta el diagnóstico y la identificación correcta del agente etiológico, de ahí que existan resultados contradictorios respecto a la incidencia y severidad de las IRA cuando coexisten infecciones virales. ${ }^{12-14}$

Por lo anterior, se planteó establecer la incidencia de coinfecciones virales en pacientes pediátricos con IRA, para lo cual se implementó el diagnóstico molecular mediante transcripción inversa seguida de reacción en cadena de la polimerasa en punto final (RT-PCR), previamente estandarizada en el laboratorio, la cual permitió la identificación y genotipificación de 200 aislamientos virales, así como determinar la incidencia de infecciones o coinfecciones virales, los patrones de circulación de los virus y su distribución geográfica. Las incidencias acumuladas fueron las siguientes: hMPV, 0.215; hMPV-hORUV, 0.015 y hMPV-hOPV, 0.23; hOPV, 0.42; hMPV-hORUV-hOPV, 0.035; hORUV-hMPV, 0.005; hORUV, 0.01. Con estos datos se pudo realizar un esquema de patrones de circulación de los diferentes virus, así como de las coinfecciones virales provocadas por hOPV, hMPV y hORUV.

\section{Método}

Previa obtención de carta de consentimiento informado, entre el 30 de agosto de 2004 y el 13 de febrero de 2014 se obtuvieron 200 muestras de exudados faríngeo y nasofaríngeo de pacientes pediátricos (0 a 14 años de edad) con infección respiratoria aguda. El proyecto fue revisado y aprobado por la Comisión de Investigación y Ética de la Facultad de Medicina, Universidad Nacional Autónoma de México (dictamen de proyecto 089/2014, oficio FMED/Cl/ SPLR/134/2014).

A partir de los aislamientos virales obtenidos de las muestras clínicas se realizó la identificación y determinación del grupo y subgrupo viral mediante oligonucleótidos diseñados en el laboratorio (Tabla 1). Para la amplificación del gen viral de interés se utilizó un termociclador (iCycler ${ }^{\mathrm{TM}}$, Bio-Rad, California, Estados Unidos) con los parámetros que se observan en la Figura 1, donde $X$ representa la temperatura de alineamiento específica de cada oligonucleótido. La fase 2 se repite 35 ciclos.

Se utilizó el programa SPSS versión 19 para el análisis estadístico de los datos. Las muestras se consideraron válidas cuando de dispuso de toda la información: cuadro clínico, historia clínica y diagnóstico del paciente, estado de procedencia de la muestra y fecha de la obtención, identificación de infección o coinfección viral. Las muestras en las que no se 


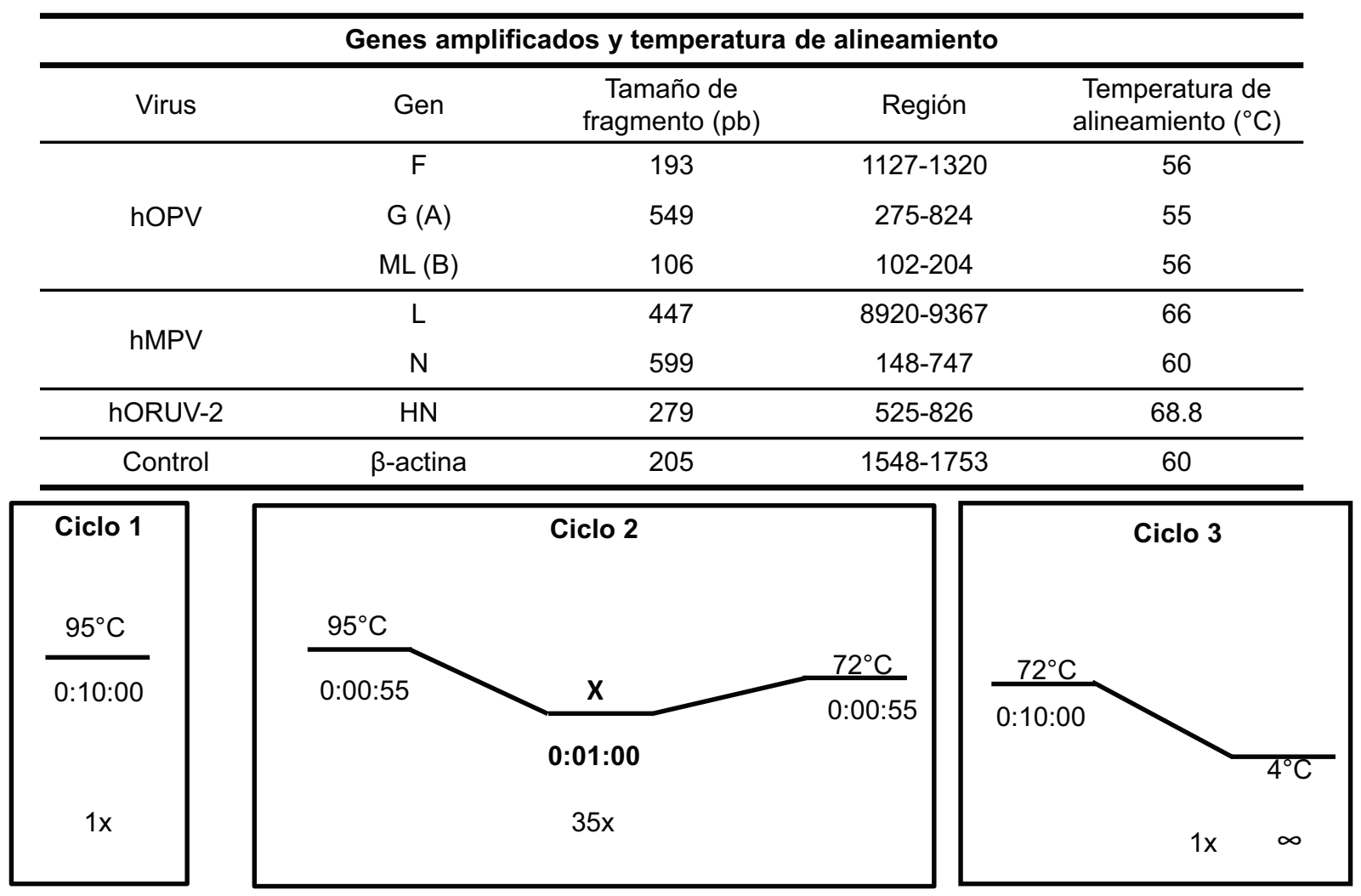

Figura 1. Amplificación de los genes virales. Ciclo 1. Desnaturalización del CADN para cada uno de los virus. Ciclo 2. Amplificación de la región seleccionada y flanqueada por cada par de oligonucleótidos. La X representa la temperatura de alineamiento de cada par de oligonucleótidos seleccionados para cada secuencia de los genes virales. La última fase de este ciclo corresponde al tiempo de extensión; este ciclo se repite 35 veces (35x). Ciclo 3. Corresponde a un ciclo extra de la fase de extensión del oligonucleótido sobre su hebra templada. La última fase es de mantenimiento del producto de amplificación. hOPV = Orthopneumovirus humano, hMPV = metapneumovirus humano, hORUV= Orthorubulavirus humano tipo 2.

Tabla 1. Secuencias de oligonucleótidos para la genotipificación de los aislamientos virales obtenidos a partir de muestras clínicas de niños con infección respiratoria aguda

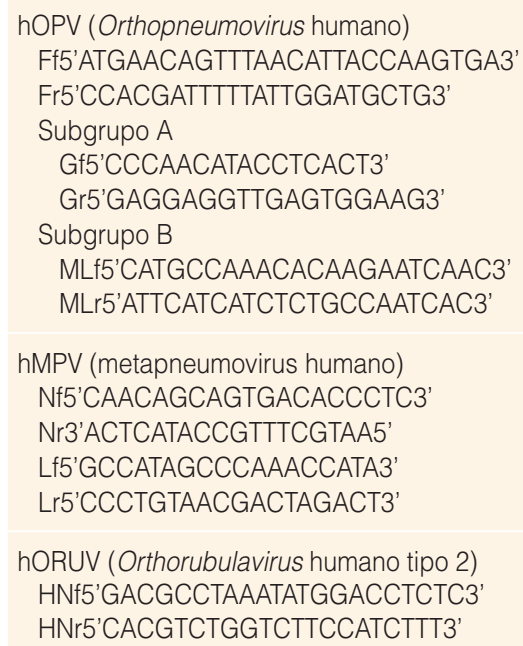

hORUV (Orthorubulavirus humano tipo 2) HNf5'GACGCCTAAATATGGACCTCTC3' HNr5'CACGTCTGGTCTTCCATCTTT3'

hOPV. Amplificación de la región 1127-1320 del gen $\mathrm{F}(\mathrm{Ff}=$ sentido, $\mathrm{Fr}=$ antisentido). Subgrupo A. Amplificación de la región 275-524 del gen G (Gf = sentido,

$\mathrm{Gr}=$ antisentido). Subgrupo B. Amplificación de la región 102-204 del gen ML (MLf = sentido, MLr = antisentido).

hMPV. Amplificación de la región 148-747 del gen $\mathrm{N}(\mathrm{Nf}=$ sentido, $\mathrm{Nr}=$ antisentido) $y$ amplificación de la región 8920-9367 del gen L ( Lf = sentido, Lr = antisentido).

hORUV. Amplificación de la región 525-826 del gen $\mathrm{HN}$ ( $\mathrm{HNf}=$ sentido, $\mathrm{HNr}=$ antisentido). disponía de alguno de estos datos se consideraron como elementos "perdidos".

\section{Resultados}

\section{Tipificación viral e incidencia acumulada de las coinfecciones virales}

Los aislamientos virales se genotipificaron por RT-PCR de punto final; de un total de 200, 186 fueron positivos para uno o varios de los tres virus estudiados y 14, negativas. En la población infantil estudiada, las incidencias acumuladas de hOPV y hMPV fueron de 0.42 y 0.215 (84 y 43 muestras positivas, respectivamente). Respecto a la incidencia acumulada de las coinfecciones virales es importante resaltar que la coinfección hMPV-hOPV subgrupo B constituyó la de mayor incidencia, con 24 casos positivos. Si se considera la coinfección por dos o más virus o subgrupos virales (hOPV, hMPV y hORUV) y no el subtipo o el gen amplificado, la coinfección por hMPV-hOPV fue la 
Tabla 2. Tipificación e incidencia acumulada de infecciones y coinfecciones virales por hOPV, hMPV y hORUV a partir de 200 muestras clínicas de niños con infección respiratoria aguda

\begin{tabular}{|l|c|c|c|c|c|c|c|}
\hline hOPV & hMPV & hORUV & hOPV-hMPV & hOPV-hORUV & hMPV-hORUV & hOPV-hMPV-hORUV & Sin identificar \\
\hline $84^{*}$ & $43^{*}$ & $2^{*}$ & $46^{*}$ & $1^{*}$ & $3^{*}$ & $7^{*}$ & $14^{*}$ \\
\hline $0.42^{* *}$ & $0.215^{\star *}$ & $0.01^{* *}$ & $0.23^{* *}$ & $0.005^{* *}$ & $0.0015^{* *}$ & $0.0035^{\star *}$ &
\end{tabular}

*Muestras positivas tipificadas por transcripción inversa seguida de reacción en cadena de la polimerasa en punto final (RT-PCR). **Incidencia acumulada de infecciones y

coinfecciones virales en niños con infección respiratoria aguda. hOPV = Orthopneumovirus humano, hMPV = metapneumovirus humano, hORUV = Orthorubulavirus humano tipo 2

de mayor incidencia, con 47 casos positivos (23\%), en contraste con las coinfecciones por hOPV-hORUV, hMPV-hORUV, que correspondieron a dos casos positivos cada una (1\%), y hOPV-hORUV-hMPV, con cinco casos positivos (2.5\%) (Tabla 2).

Las muestras clínicas positivas a hOPV por la amplificación del fragmento correspondiente al gen $F$, se subtipificaron en los subgrupos A y B. Para el subgrupo $A$ se amplificó un fragmento del gen que codifica para la glucoproteína $G$ y para el subgrupo $B$, un fragmento del gen que codifica para las proteínas $M$ y L. Nuestros resultados mostraron que de las 40 muestras positivas a hOPV, $48 \%$ correspondió al subgrupo $B$, mientras que $27 \%$ al subgrupo $A$ y $3 \%$ a coinfecciones por ambos subgrupos. En $22 \%$ de las muestras positivas a hOPV no fue posible identificar el subgrupo viral, lo que pudo deberse a probables mutaciones en las regiones amplificadas del subgrupo A o B (Figura 2).

\section{Patrones de circulación y su distribución en el tiempo}

En México no se dispone de patrones de circulación de virus respiratorios, incidencia de coinfecciones y distribución geográfica. Además de las implicaciones en la severidad del cuadro clínico, es importante definir la incidencia de los patrones de circulación en función de la estacionalidad. Para esa razón se determinó la fecha de la obtención, la cronología de circulación y el lugar de procedencia de las 136 muestras que contaban con esa información. Los resultados de la incidencia acumulada demostraron que el mayor número de casos positivos de virus circulantes o cocirculantes se presentó de enero a marzo (Figura 3), lo cual coincide con la estacionalidad de estos virus; sin embargo, fue posible identificar virus circulando o cocirculando entre abril y septiembre, periodo que corresponde a la época de lluvia en México, si bien es cierto que la incidencia acumulada fue menor, lo cual coincide con reportes de otros países.

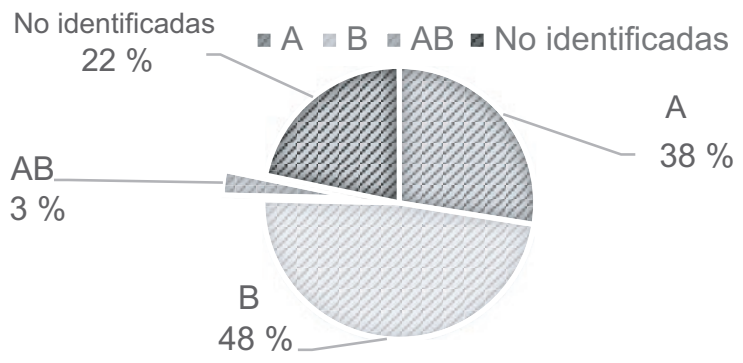

Figura 2. Tipificación de los aislamientos virales en los subgrupos $A$ y $B$ de Orthopneumovirus humano; se realizó mediante RT-PCR (transcripción inversa seguida de reacción en cadena de la polimerasa) en punto final y los productos de amplificación se observaron en gel de agarosa teñido con Gelred. Los productos de amplificación se procesaron y analizaron con el programa Quantity One Bio-Rad de ChemiDoc Hood II.

\section{Mapas geográficos de las coinfecciones virales según la incidencia acumulada}

La determinación de la incidencia acumulada permitió trazar los mapas geográficos relacionados con los patrones de circulación de la población en estudio y determinar el flujo de los virus respiratorios en diferentes estados de México (Figura 4). De igual forma se estableció el patrón de circulación geográfica en los aislamientos virales positivos a hOPV, subtipificados en A o B (Figura 5).

\section{Discusión}

En este proyecto se analizaron 200 muestras clínicas de pacientes pediátricos con infección respiratoria aguda. Nuestra investigación es un estudio piloto transversal prospectivo observacional, el cual abarcó del 30 de agosto de 2004 al 13 de febrero de 2014, cada muestra fue obtenida con la autorización por escrito y previa información de los padres o tutores. Se determinó la incidencia acumulada, medida del índice dinámico asociada a la aparición de nuevos "casos" en el tiempo y en la población seleccionada. A partir de 200 aislamientos virales se definió la 


\begin{tabular}{|c|c|c|c|}
\hline wOPV $(\mathrm{F})(\mathrm{A})$ & a hMPV $(\mathrm{N}$ y L $)$ y hORUV & InMPV ( $\mathrm{N}$ y L $)$ & $\llbracket \mathrm{hMPV}(\mathrm{N})$ y hOPV $(\mathrm{B})$ y hOPV $(\mathrm{F})$ \\
\hline hOPV $(\mathrm{A})$ & a hOPV $(\mathrm{F})(\mathrm{A}$ y B $)$ y hMPV $(\mathrm{N})$ & Negativas para tres virus & ahOPV $(\mathrm{F})$ \\
\hline ahORUV & $\mathrm{hOPV}(\mathrm{F})(\mathrm{Ay} \mathrm{B})$ & a hOPV $(\mathrm{F})(\mathrm{B})$ & a hORUV y hOPV F) y hMPV (N) \\
\hline mMPV $(\mathrm{L})$ & hMPV (N y L) y hOPV (B) & a hOPV (F y B) y hMPV (N y L) & hORUV y hOPV(F y B) y hMPV (N) \\
\hline WOPV $(\mathrm{F}$ y B $)$ y hORUV & \multicolumn{2}{|c|}{ a hORUV y hOPV $(\mathrm{F}$ y A) y hMPV $(\mathrm{N})$ hOPV $(\mathrm{F})$ y hMPV $(\mathrm{L})$} & 国hMPV $(\mathrm{L})$ y hOPV $(\mathrm{B})$ \\
\hline
\end{tabular}

40

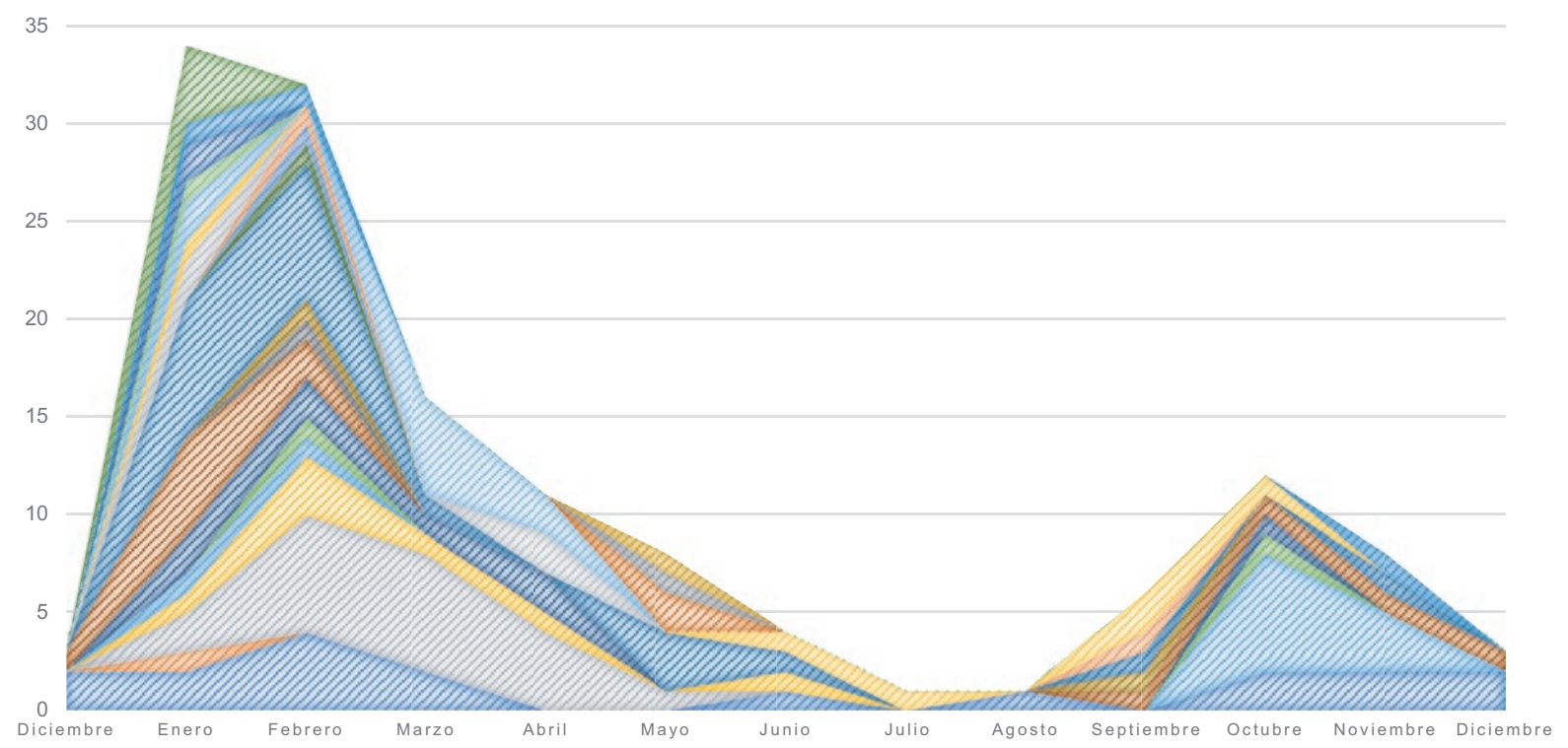

Figura 3. Incidencia acumulada y su relación con la estacionalidad climática. Se determinó la incidencia acumulada de los casos tipificados como positivos para cada una de las infecciones y coinfecciones virales y se relacionó con la fecha de la obtención de la muestra para establecer los patrones de circulación y su estacionalidad climática. hOPV = Orthopneumovirus humano, hMPV = metapneumovirus humano, hORUV = Orthorubulavirus humano tipo 2.

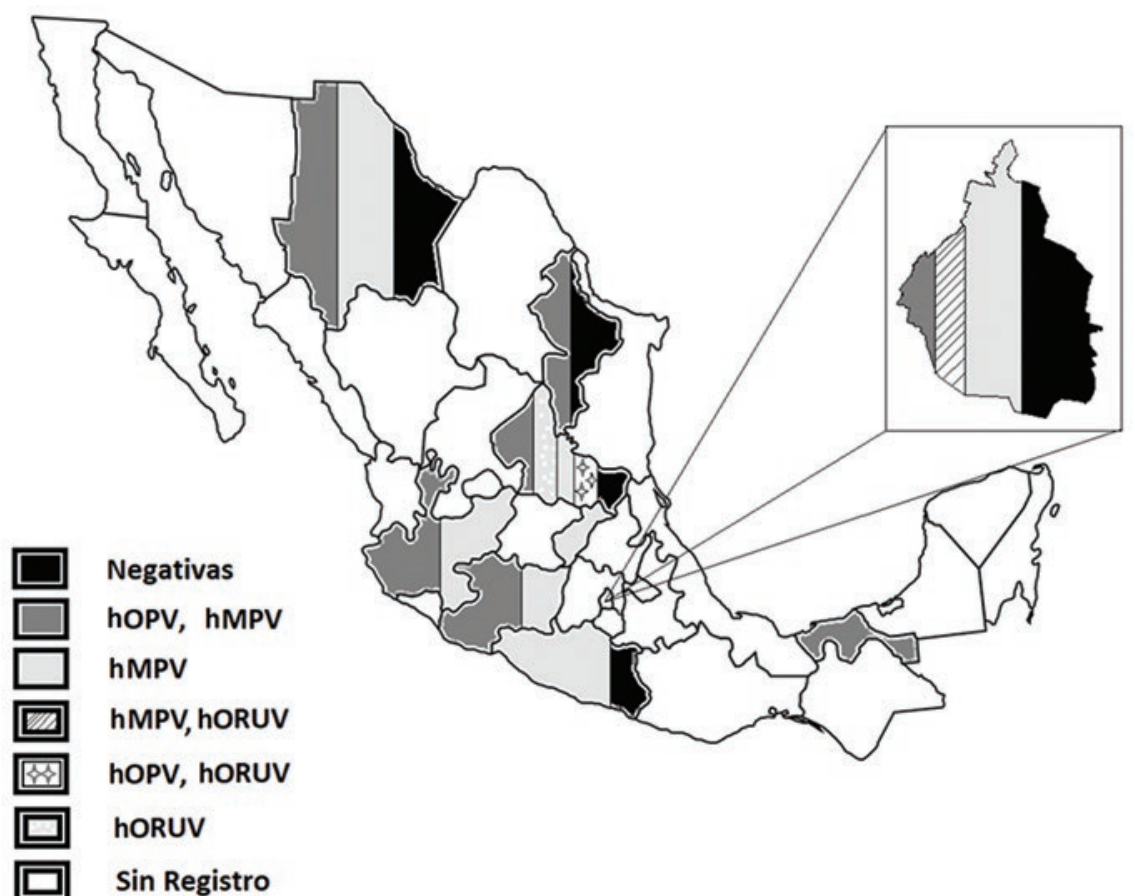

Figura 4. Distribución geográfica de las infecciones y coinfecciones virales en México a partir de aislamientos virales obtenidos de 200 muestras de exudados faríngeo y nasofaríngeo de pacientes pediátricos. hOPV = Orthopneumovirus humano, hMPV = metapneumovirus humano, hORUV = Orthorubulavirus humano tipo 2. 


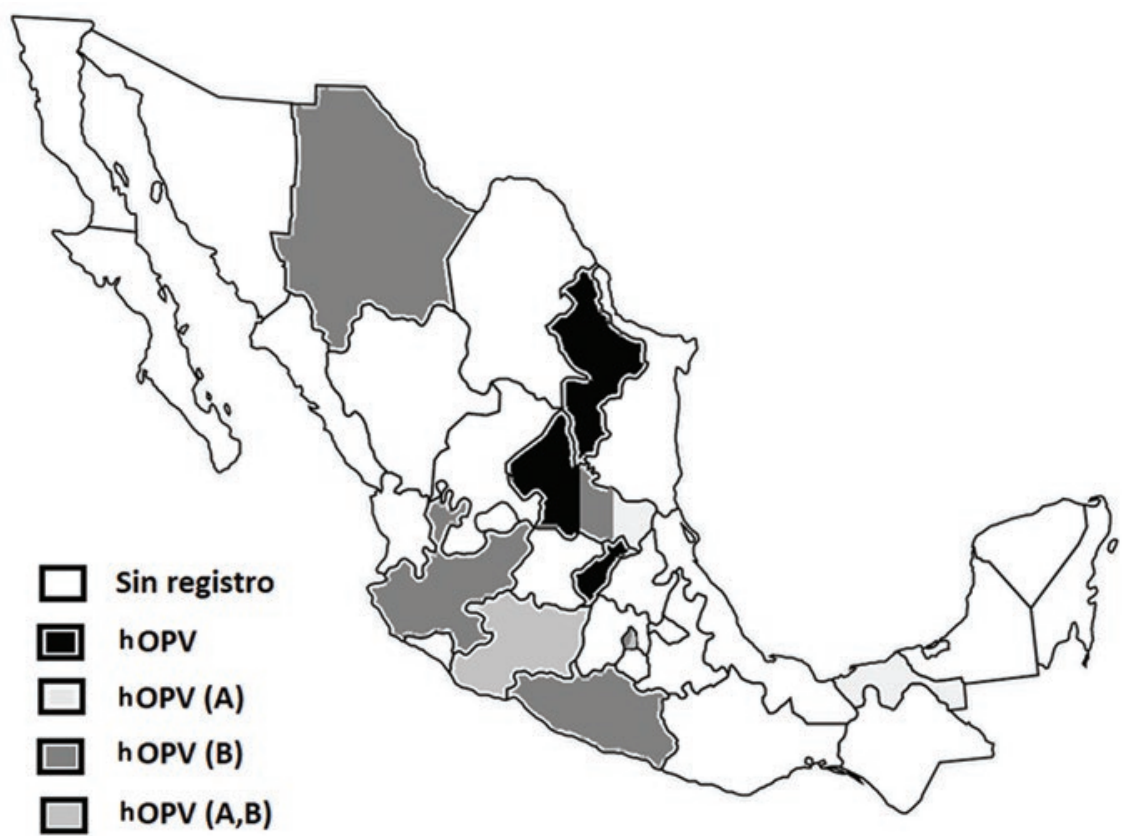

Figura 5. Distribución geográfica en México de los subgrupos virales de los casos positivos a hOPV determinada a partir de los datos de la incidencia acumulada. Los aislamientos virales tipificados como hOPV se subtipificaron en $A$ y B. hOPV = Orthopneumovirus humano, hMPV = metapneumovirus humano, hORUV = Orthorubulavirus humano tipo 2.

incidencia acumulada de las infecciones y coinfecciones por hOPV, hMPV y hORUV; con esos datos se trazaron los patrones de circulación y la distribución geográfica respecto a la estacionalidad. Los datos de incidencia acumulada para las infecciones asociadas a un solo virus demostraron que el más frecuente fue hOPV, con incidencia de $0.42,48 \%$ correspondió al subtipo $B$ y $38 \%$, al subtipo $A$. Este resultado coincide con los reportes de prevalencia del subgrupo $B$ en diferentes poblaciones de China durante los brotes de 2009-2010, 2012-2013 y 2006-2014, ${ }^{15,16}$ del cual BA9 y GB5 fueron los de mayor circulación. ${ }^{16,17}$

También fue posible trazar la distribución geográfica: la Ciudad de México, Chihuahua, San Luis Potosí, Jalisco y Guerrero presentaron el mayor número de casos positivos para hOPV subgrupo B. La incidencia acumulada y la estacionalidad de los subgrupos A y $B$ en Chihuahua y San Luis Potosí pueden estar relacionadas con el periodo invernal y los frentes fríos; en Jalisco y Guerrero, el incremento en el número de casos se asocia con el periodo de lluvia, que se produce por las corrientes húmedas de los vientos alisios de ambos hemisferios en la llamada "zona intertropical de convergencia". Este patrón en la estacionalidad se repitió en los otros dos virus en estudio, no obstante, la distribución geográfica de hMPV fue más amplia: se identificaron muestras en Ciudad de México, Chihuahua, San Luis Potosí, Querétaro, Jalisco,
Michoacán y Guerrero. Los patrones de circulación de estos virus durante la temporada invernal y el periodo de lluvia pueden asociarse al efecto del clima en los mecanismos de defensa inmune innata en el sistema respiratorio, como disminución del movimiento mucociliar y deficiencia en la capacidad de las vías respiratorias superiores para calentar adecuadamente el aire frío, lo que favorece este tipo de infecciones. Durante la temporada de lluvia, la humedad del medio ambiente contribuye a que las gotas de saliva se mantengan en suspensión por más tiempo, lo que facilita su propagación.

Nuestros datos coinciden con el reporte del predominio de los rinovirus tipos $\mathrm{A}$ y $\mathrm{C}$ durante la temporada de lluvia y principios del invierno. ${ }^{18}$ Además, un estudio retrospectivo realizado entre 1985 y 1987 en niños de Nigeria con infección respiratoria mostró que el pico de incidencia de los casos reportados se presentó en septiembre, en la temporada de lluvia, a la que le sigue la temporada de los vientos alisios en África Occidental, caracterizada por frío, sequedad con polvo y baja humedad durante los meses de noviembre a marzo (harmatán), condiciones que dañan las vías respiratorias y favorecen la exposición a infecciones. ${ }^{19-21}$ Nuestros resultados también concuerdan con el señalamiento de que los coronavirus respiratorios humanos (HCoV) son responsables de IRA durante el harmatán y la época de lluvia. ${ }^{21-23}$ 
Nuestros hallazgos demostraron que la mayor incidencia acumulada de coinfecciones virales se presentó en San Luis Potosí (tres casos) y la Ciudad de México (dos casos); además, San Luis Potosí fue el único estado en el que se aislaron e identificaron muestras positivas a hORUV como único agente causal y en coinfección con hOPV y hMPV. Nuestra investigación permitió detectar coinfecciones virales también en Chihuahua, Nuevo León, Jalisco, Michoacán y Tabasco. La cocirculación de estos virus respiratorios, su coincidencia estacional y las condiciones del sistema inmune de los pacientes infectados favorecen las infecciones múltiples. En otro estudio de nuestro grupo de trabajo se concluyó que las coinfecciones virales modifican el cuadro clínico respiratorio, provocando manifestaciones "atípicas" con la aparición de signos y síntomas como fiebre, dolor abdominal, estridor laríngeo y rinorrea hialina, lo cual dificulta el diagnóstico y la identificación correcta del agente etiológico. ${ }^{12}$

Finalmente, nuestros resultados sugieren que la edad pediátrica es un factor predisponente para la infección viral, probablemente debido a que la maduración pulmonar se logra después de los ocho años. ${ }^{12}$ En México no se dispone de datos epidemiológicos, probablemente por falta de información en el expediente clínico (documento médico-legal), que recopila el orden cronológico de los padecimientos del paciente y contribuye al diagnóstico eficiente. Sin esa información, el diagnóstico se vuelve azaroso y se pierden de vista los factores que determinan un padecimiento o las circunstancias que facilitan el diagnóstico preciso:12 además, se pierde la oportunidad de determinar los patrones de circulación de los virus.

Investigaciones recientes abordan las coinfecciones virales y los nuevos síntomas respiratorios, así como nuevas estrategias de salud para hacer frente a las infecciones y disminuir las tasas de mortalidad en los niños. ${ }^{12}$ No obstante, el sesgo por el número de muestras analizadas, la incidencia reportada de cada virus y las coinfecciones asociadas proporcionaron los datos necesarios para realizar un esquema de patrones de circulación y la distribución geográfica en México.

\section{Agradecimientos}

Nuestro agradecimiento al Hospital Regional "Adolfo López Mateos", Facultad de Medicina de la Universidad Autónoma de San Luis Potosí e Instituto de Diagnóstico y Referencia Epidemiológicos, por la donación de las muestras clínicas. A Antonio Magaña Hernández, por sus observaciones al manuscrito.

\section{Conflicto de intereses}

Los autores declaran no tener conflicto de intereses.

\section{Financiamiento}

Proyecto apoyado por la Dirección General de Apoyo al Personal Académico de la Universidad Nacional Autónoma de México (IN224316; PE211115).

\section{Responsabilidades éticas}

Protección de personas y animales. Los autores declaran que para esta investigación no se realizaron experimentos en seres humanos ni en animales.

Confidencialidad de los datos. Los autores declaran que en este artículo no aparecen datos de pacientes.

Derecho a la privacidad y consentimiento informado. Los autores obtuvieron el consentimiento informado de los tutores de los niños referidos en el artículo. El documento obra en poder del autor de correspondencia.

\section{Bibliografía}

1. Organización Mundial de la Salud [Internet]. Suiza: Neumonía; 2019.

2. Knipe D, Howley P. Fields virology. Volumen 2. EE. UU.: Lippincott Williams \& Wilkins: 2007.

3. Hall CB, Simőes EA, Anderson LJ. Clinical and epidemiologic features of respiratory syncytial virus. Curr Top Microbiol Immunol. 2013:372:39-57

4. Wertz GW, Moudy RM. Antigenic and genetic variation in human respiratory syncytial virus. Pediatr Infect Dis J. 2004;23:19-24.

5. Bezerra PGM, Britto MCA, Correia JB, Duarte MC, Fonceca AM, Rose K, et al. Viral and atypical bacterial detection in acute respiratory infection in children under five years. PLoS One. 2011;6:4-12.

6. García-García ML, Calvo C, Rey C, Díaz B, Del Mar-Molinero M, Pozo F, et al. Human metapneumovirus infections in hospitalized children and comparison with other respiratory viruses. 2005-2014 prospective study. PLoS One. 2017:12:e0173504.

7. Price RHM, Graham C, Ramalingam S. Association between viral seasonality and meteorological factors. Sci Rep. 2019;9:1-11.

8. Manzoni P, Figueras-Aloy J, Simões EAF, Checchia PA, Fauroux B, Bont $L$, et al. Defining the incidence and associated morbidity and mortality of severe respiratory syncytial virus infection among children with chronic diseases. Infect Dis Ther. 2017;6:383-411.

9. Secretaría de Salud. Anuarios de morbilidad 1984-2018. México: SSa; 2020.

10. Wong-Chew RM, García-León ML, Noyola DE, Pérez-González LF, Gaitán-Meza J, Villaseñor-Sierra A, et al. Respiratory viruses detected in Mexican children younger than 5 years old with community-acquired pneumonia: A national multicenter study. Int J Infect Dis. 2017:62:32-38.

11. Instituto Mexicano del Seguro Social. Guía de práctica clínica de diagnóstico y tratamiento de bronquiolitis aguda en niñas/niños y en el primer nivel de atención. México: Instituto Mexicano del Seguro Social/Secretaría de Salud: 2015.

12. Hernández D. Estudio piloto de infecciones respiratorias agudas en coinfecciones virales (virus sincitial respiratorio y metapneumovirus humano) y su impacto en las manifestaciones clínicas, diagnósticas y epidemiológicas. Contacto Químico. 2016;16:11-13.

13. Robledo-Aceves M, Moreno-Peregrina MJ, Velarde-Rivera F, Ascencio-Esparza E, Preciado-Figueroa F, Escobedo-Meléndez G, et al. Risk factors for severe bronchiolitis caused by respiratory virus infections among Mexican children in an emergency department. Medicine (Baltimore). 2018;97:9. 
14. Díaz J, Morales-Romero J, Pérez-Gil G, Bedolla-Barajas M, Delgado-Figueroa N, García-Román R, et al. Viral coinfection in acute respiratory infection in Mexican children treated by the emergency service: A cross-sectional study. Ital J Pediatr. 2015;4:33.

15. Ren L, Xiao Q, Zhou L, Xia Q, Liu E. Molecular characterization of human respiratory syncytial virus subtype $B$ : a novel genotype of subtype $B$ circulating in China. J Med Virol. 2015;87:1-9.

16. Song J, Wang $\mathrm{H}$, Shi J, Cui A, Huang $Y$, Sun L, et al. Emergence of BA9 genotype of human respiratory syncytial virus subgroup $B$ in China from 2006 to 2014. Sci Rep. 2017;7:16765.

17. Fan R, Fan C, Zhang J, Wen B, Lei Y, Liu C, et al. Respiratory syncytial virus subtype ON1/NA1/BA9 predominates in hospitalized children with lower respiratory tract infections. J Med Virol. 2017;89:213-221.

18. Matthew J, Pinto-Pereira LM, Pappas TE, Swenson CA, Grindle KA Roberg KA, et al. Distribution and seasonality of rhinovirus and other respiratory viruses in a cross-section of asthmatic children in Trinidad, West Indies. Ital J Pediatr. 2009;35:1-10.
19. Olaleye OD, Omilabu SA, Olabode AO, Fagbami AH. Serological evidence for influenza virus activity in Nigeria (1985-1987). Virologie. 1989;40:11-17.

20. Maïnassara HB, Lagare A, Tempia S, Sidiki A, Issaka B, Abdou Sidikou $B$, et al. Influenza sentinel surveillance among patients with influenza-like-illness and severe acute respiratory illness within the framework of the National Reference Laboratory, Niger, 2009-2013. PLoS One. 2015;10:1-9.

21. Owusu M, Annan A, Corman VM, Larbi R, Anti P, Drexler JF, et al. Human coronaviruses associated with upper respiratory tract infections in three rural areas of Ghana. PLoS One. 2014;9:24-27.

22. Talla-Nzussouo N, Duque J, Adedeji AA, Coulibaly D, Sow S, Tarnagda Z, et al. Epidemiology of influenza in West Africa after the 2009 influenza A(H1N1) pandemic, 2010-2012. BMC Infect Dis. 2017;17:1-8.

23. Dia N, Sarr FD, Thiam D, Sarr TF, Espié E, OmarBa I, et al. Influenza-like illnesses in Senegal: not only focus on influenza viruses. PLoS One. 2014;9:e93227. 